HIP-1997-26/TH

hep-ph/9708486

\title{
Mass of the lightest Higgs Boson in Supersymmetric Left-Right Models
}

\author{
K. Huitu ${ }^{a}$, P.N. Pandita ${ }^{a, b}$ and K. Puolamäki ${ }^{a}$ \\ ${ }^{a}$ Helsinki Institute of Physics, P.O.Box 9, FIN-00014 University of Helsinki, \\ Finland \\ ${ }^{b}$ Department of Physics, North Eastern Hill University, Shillong 793022, India凹
}

\begin{abstract}
We consider the lightest Higgs boson in naturally R-parity conserving supersymmetric left-right models. We obtain an upper bound on the tree level mass of this lightest Higgs boson. This upper bound depends on the $S U(2)_{L}$ and $S U(2)_{R}$ gauge couplings, and the vacuum expectation values of bidoublet Higgs fields, which are needed to break $S U(2)_{L} \times U(1)_{Y}$. The upper bound does not depend on either the $S U(2)_{R}$ breaking scale or the supersymmetry breaking scale. We evaluate the bound numerically by assuming that the theory remains perturbative upto some scale $\Lambda$. We find that the bound can be considerably larger than in MSSM. The dominant radiative corrections to the upper bound due to top-stop and bottom-sbottom systems are of the same form as in the minimal supersymmetric standard model.
\end{abstract}

PACS numbers: 12.60.Jv, 12.60.Cn, 14.80.Cp

\footnotetext{
${ }^{1}$ Permanent address
} 
Considerable importance attaches to the study of the Higgs bosons of the minimal supersymmetric standard model (MSSM), based on the Standard Model gauge group $S U(2)_{L} \times U(1)_{Y}$, with two Higgs doublet superfields [1]. It is well known that, because of underlying gauge invariance and supersymmetry (SUSY), the lightest Higgs boson of MSSM has a tree level upper bound of $m_{Z}$ (the mass of $\mathrm{Z}$ boson) on its mass [2]. Although radiative corrections [3] to the tree level result can be appreciable, these depend only logarithmically on the SUSY breaking scale, and are, therefore, under control. This results in an upper bound of about $125-135 \mathrm{GeV}$ on the radiatively corrected mass of the lightest Higgs boson of MSSM [4]. Because of the presence of additional trilinear Yukawa couplings, such a tight constraint on the mass of the lightest Higgs boson need not a priori hold in extensions of MSSM with an extended Higgs sector. Nevertheless, it has been shown that the upper bound on the lightest Higgs boson mass in these models depends only on the weak scale and dimensionless coupling constants (and only logarithmically on SUSY breaking scale), and is calculable if all the couplings remain perturbative below some scale $\Lambda$ [5-11]. This upper bound can vary between $150 \mathrm{GeV}$ to $165 \mathrm{GeV}$ depending on the Higgs structure of the supersymmetric model. Thus, nonobservation of a light Higgs boson below this upper bound will rule out an entire class of supersymmetric models based on the gauge group $S U(2)_{L} \times U(1)_{Y}$.

The existence of the upper bound on the lightest Higgs boson mass in MSSM with arbitrary Higgs sectors has been investigated in a situation where the underlying supersymmetric model respects the discrete matter parity, or R-parity $\left(R_{P}\right)$ symmetry [12, 13], under which all Standard Model particles are even and their superpartners are odd, so that all renormalizable baryon (B) and lepton (L) number violating terms in the superpotential are forbidden. However, the assumption of R-parity conservation appears to be $a d$ hoc, since it is not required for the internal consistency of the minimal supersymmetric standard model. Furthermore, all global symmetries, discrete or continuous, could be violated by the Planck scale physics effects [14]. The problem becomes accute for low energy supersymmetric models [15], because B and L are no longer automatic symmetries of the Lagrangian as they are in the Standard Model.

It would, therefore, be more appealing to have a supersymmetric theory where 
R-parity is related to a gauge symmetry, and its conservation is automatic because of the invariance of the underlying theory under an extended gauge symmetry. Indeed $R_{P}$ conservation follows automatically in certain theories with gauged $(B-L)$, as is suggested by the fact that matter parity is simply a $Z_{2}$ subgroup of $(B-L)$. It has been noted by several authors [16, 17] that if the gauge symmetry of MSSM is extended to $S U(2)_{L} \times U(1)_{I_{3 R}} \times U(1)_{B-L}$, or $S U(2)_{L} \times S U(2)_{R} \times U(1)_{B-L}$, the theory becomes automatically R-parity conserving. Such a supersymmetric leftright theory (SUSYLR) solves the problems of explicit B and L violation of MSSM, and has received much attention recently [18-23].

Since such a naturally R-parity conserving theory necessarily involves the extension of the Standard Model gauge group, and since the extended gauge symmetry has to be broken, it involves a new scale, the scale of left-right symmetry breaking, beyond the SUSY and $S U(2)_{L} \times U(1)_{Y}$ breaking scales of MSSM. It is, therefore, important to ask whether the upper bound on the lightest Higgs mass in naturally R-parity conserving theories depends on the scale of the breakdown of the extended gauge group. In this paper we investigate the Higgs sector of the supersymmetric left-right theory in order to answer this question. We find that the tree level upper bound on the lightest Higgs boson mass does not explicitly depend on the scale of the left-right symmetry breaking. It depends on the scale of the $S U(2)_{L} \times U(1)_{Y}$ breaking and dimensionless coupling constants only. We then calculate the dominant one-loop radiative corrections due to top-stop and bottom-sbottom to this upper bound on the lightest Higgs mass in the supersymmetric left-right models. These turn out to be of the same order of magnitude as the corresponding radiative corrections in models based on $S U(2)_{L} \times U(1)_{Y}$. Although the R-parity is conserved at the level of Lagrangian, it is necessarily spontaneously broken in this class of models [20]. We will see that the upper bound does not depend on the VEV of the right-handed sneutrino responsible for the spontaneous R-parity violation.

We begin by recalling the basic features of the left-right supersymmetric models. The quark and lepton doublets are included in $Q(2,1,1 / 3) ; Q^{c}(1,2,-1 / 3)$; $L(2,1,-1) ; L^{c}(1,2,1)$, where $Q$ and $Q^{c}$ denote the left- and right-handed quark superfields and similarly for the leptons $L$ and $L^{c}$. The Higgs superfields consist of $\Delta_{L}(3,1,-2) ; \Delta_{R}(1,3,-2) ; \delta_{L}(3,1,2) ; \delta_{R}(1,3,2) ; \Phi(2,2,0) ; \chi(2,2,0)$. The numbers 
in the parentheses denote the representation content of the fields under the gauge group $S U(2)_{L} \times S U(2)_{R} \times U(1)_{B-L}$. We note that two $S U(2)_{R}$ Higgs triplet superfields $\Delta_{R}(1,3,-2)$ and $\delta_{R}(1,3,2)$ with opposite $(B-L)$ are necessary to break the left-right symmetry spontaneously, and to cancel triangle gauge anomalies due to the fermionic superpartners. The left-right model also contains the $S U(2)_{L}$ triplets $\Delta_{L}$ and $\delta_{L}$ in order to make the Lagrangian fully symmetric under the $L \leftrightarrow R$ transformation, although these are not needed phenomenologically for the symmetry breaking or the see-saw mechanism.

We further note that there are two bidoublet Higgs superfields $\Phi$ and $\chi$ in order to break the $S U(2)_{L} \times U(1)_{Y}$ and to generate a nontrivial Kobayashi-Maskawa matrix. The most general gauge invariant superpotential involving these superfields can be written as

$$
\begin{aligned}
W= & h_{\phi Q} Q^{T} i \tau_{2} \Phi Q^{c}+h_{\chi Q} Q^{T} i \tau_{2} \chi Q^{c}+h_{\phi L} L^{T} i \tau_{2} \Phi L^{c}+h_{\chi L} L^{T} i \tau_{2} \chi L^{c} \\
& +h_{\delta_{L}} L^{T} i \tau_{2} \delta_{L} L+h_{\Delta_{R}} L^{c T} i \tau_{2} \Delta_{R} L^{c}+\mu_{1} \operatorname{Tr}\left(i \tau_{2} \Phi^{T} i \tau_{2} \chi\right)+\mu_{1}^{\prime} \operatorname{Tr}\left(i \tau_{2} \Phi^{T} i \tau_{2} \Phi\right) \\
& +\mu_{1}^{\prime \prime} \operatorname{Tr}\left(i \tau_{2} \chi^{T} i \tau_{2} \chi\right)+\operatorname{Tr}\left(\mu_{2 L} \Delta_{L} \delta_{L}+\mu_{2 R} \Delta_{R} \delta_{R}\right) .
\end{aligned}
$$

The general form of the Higgs potential is given by

$$
V=V_{F}+V_{D}+V_{s o f t}
$$

which can be calculated in a straightforward manner. In the following we shall represent the scalar components of the Higgs superfields by the same symbols as the superfields themselves and add a tilde on the scalar components of lepton and quark superfields. The most general form of the vacuum expectation values of various scalar fields, which preserves $U(1)_{e m}$ can be written as

$$
\begin{aligned}
& \langle\Phi\rangle=\left(\begin{array}{cc}
\kappa_{1} & 0 \\
0 & e^{i \varphi_{1}} \kappa_{1}^{\prime}
\end{array}\right),\langle\chi\rangle=\left(\begin{array}{cc}
e^{i \varphi_{2}} \kappa_{2}^{\prime} & 0 \\
0 & \kappa_{2}
\end{array}\right), \\
& \left\langle\Delta_{L}\right\rangle=\left(\begin{array}{cc}
0 & v_{\Delta_{L}} \\
0 & 0
\end{array}\right),\left\langle\delta_{L}\right\rangle=\left(\begin{array}{cc}
0 & 0 \\
v_{\delta_{L}} & 0
\end{array}\right), \\
& \left\langle\Delta_{R}\right\rangle=\left(\begin{array}{cc}
0 & v_{\Delta_{R}} \\
0 & 0
\end{array}\right),\left\langle\delta_{R}\right\rangle=\left(\begin{array}{cc}
0 & 0 \\
v_{\delta_{R}} & 0
\end{array}\right),
\end{aligned}
$$




$$
\langle L\rangle=\left(\begin{array}{c}
\sigma_{L} \\
0
\end{array}\right),\left\langle L^{c}\right\rangle=\left(\begin{array}{c}
0 \\
\sigma_{R}
\end{array}\right) .
$$

We note that the triplet vacuum expectation values $v_{\Delta_{R}}$ and $v_{\delta_{R}}$ represent the scale of $S U(2)_{R}$ breaking and are, therefore, assumed to be large, in the range $v_{\Delta_{R}}, v_{\delta_{R}} \gtrsim 1 \mathrm{TeV}$. These represent a new scale of physics, the right-handed scale which we shall generically denote as $v_{R}$. Since the mixing between the charged gauge bosons is tiny, and to avoid the flavor changing neutral currents, $\kappa_{1}^{\prime}$ and $\kappa_{2}^{\prime}$ are taken to be much smaller than $\kappa_{1}$ and $\kappa_{2}$, and we shall ignore them in the following. Furthermore, since the electroweak $\rho$-parameter is close to unity, $\rho=1.002 \pm 0.0013 \pm 0.0018$ [24], the triplet vacuum expectation values $\left\langle\Delta_{L}\right\rangle$ and $\left\langle\delta_{L}\right\rangle$ must be small, and we shall ignore them as well. Since the spontaneous breakdown of R-parity is inevitable [20], we shall assume that at least one of the VEVs $\langle\tilde{\nu}\rangle$ or $\left\langle\tilde{\nu}^{c}\right\rangle$ is non-zero. The $\operatorname{VEV}\langle\tilde{\nu}\rangle \equiv \sigma_{L}$ is at most of the order of the weak scale, since it contributes to lighter weak gauge boson masses. It is important to note that in $Q_{e m}$ preserving ground state $\left\langle\tilde{\nu}^{c}\right\rangle \equiv \sigma_{R}$ is necessarily at least of the order of the typical SUSY breaking scale $M_{S U S Y}$ or the right-handed breaking scale $\left(v_{\Delta_{R}}, v_{\delta_{R}}\right)$, whichever is lower [25].

We now proceed to the main point of this paper by constructing the mass matrix for the neutral scalars. To this end we write down explicitly the different components of the scalar potential (2) $\left(g_{L}, g_{R}, g_{B-L}\right.$ are the gauge couplings)

$$
\begin{aligned}
V_{F}= & +\left|h_{\phi L} i \tau_{2} \phi L^{c}+h_{\chi L} i \tau_{2} \chi L^{c}+2 h_{\delta_{L}} L^{T} i \tau_{2} \delta_{L}\right|^{2} \\
& +\left|h_{\phi L} L^{T} i \tau_{2} \phi+h_{\chi L} L^{T} i \tau_{2} \chi+2 h_{\Delta_{R}} L^{c T} i \tau_{2} \Delta_{R}\right|^{2} \\
& +\left|h_{\Delta_{R}} L^{c} L^{c T}\left(i \tau_{2}\right)+\mu_{2 R} \delta_{R}\right|^{2}+\left|h_{\delta_{L}} L L^{T}\left(i \tau_{2}\right)+\mu_{2 L} \Delta_{L}\right|^{2} \\
& +\left|h_{\phi Q} Q^{c} Q^{T}\left(i \tau_{2}\right)+h_{\phi L} L^{c} L^{T}\left(i \tau_{2}\right)+\mu_{1}\left(i \tau_{2}\right) \chi^{T}\left(i \tau_{2}\right)+2 \mu_{1}^{\prime}\left(i \tau_{2}\right) \Phi^{T}\left(i \tau_{2}\right)\right|^{2} \\
& +\left|h_{\chi Q} Q^{c} Q^{T}\left(i \tau_{2}\right)+h_{\chi L} L^{c} L^{T}\left(i \tau_{2}\right)+\mu_{1}\left(i \tau_{2}\right) \Phi^{T}\left(i \tau_{2}\right)+2 \mu_{1}^{\prime \prime}\left(i \tau_{2}\right) \chi^{T}\left(i \tau_{2}\right)\right|^{2} \\
& +\left|\left(i \tau_{2}\right)\left(h_{\phi Q} \Phi+h_{\chi Q} \chi\right) Q^{c}\right|^{2}+\left|Q^{T}\left(i \tau_{2}\right)\left(h_{\phi Q} \Phi+h_{\chi Q} \chi\right)\right|^{2} \\
& +\left|\mu_{2 R} \Delta_{R}\right|^{2}+\left|\mu_{2 L} \delta_{L}\right|^{2},
\end{aligned}
$$




$$
\begin{aligned}
V_{D}= & \frac{1}{8} g_{L}^{2} \sum_{a}\left[\operatorname{Tr}\left(\Phi^{\dagger} \tau_{a} \Phi\right)+\operatorname{Tr}\left(\chi^{\dagger} \tau_{a} \chi\right)+2 \operatorname{Tr}\left(\Delta_{L}^{\dagger} \tau_{a} \Delta_{L}\right)+2 \operatorname{Tr}\left(\delta_{L}^{\dagger} \tau_{a} \delta_{L}\right)\right. \\
& \left.+L^{\dagger} \tau_{a} L+Q^{\dagger} \tau_{a} Q\right]^{2}+\frac{1}{8} g_{R}^{2} \sum_{a}\left[-\operatorname{Tr}\left(\Phi \tau_{a} \Phi^{\dagger}\right)-\operatorname{Tr}\left(\chi \tau_{a} \chi^{\dagger}\right)\right. \\
& \left.+2 \operatorname{Tr}\left(\Delta_{R}^{\dagger} \tau_{a} \Delta_{R}\right)+2 \operatorname{Tr}\left(\delta_{R}^{\dagger} \tau_{a} \delta_{R}\right)+L^{c \dagger} \tau_{a} L^{c}+Q^{c \dagger} \tau_{a} Q^{c}\right]^{2} \\
& +\frac{1}{8} g_{B-L}^{2}\left[2 \operatorname{Tr}\left(-\Delta_{R}^{\dagger} \Delta_{R}+\delta_{R}^{\dagger} \delta_{R}-\Delta_{L}^{\dagger} \Delta_{L}+\delta_{L}^{\dagger} \delta_{L}\right)\right. \\
& \left.-L^{\dagger} L+L^{c \dagger} L^{c}+\frac{1}{3} Q^{\dagger} Q-\frac{1}{3} Q^{c \dagger} Q^{c}\right]^{2}
\end{aligned}
$$

and

$$
\begin{aligned}
V_{\text {soft }}= & m_{1}^{2} \operatorname{Tr}|\phi|^{2}+m_{2}^{2} \operatorname{Tr}|\chi|^{2}-\left(m_{\phi \chi}^{2} \operatorname{Tr}\left(i \tau_{2} \phi^{T} i \tau_{2} \chi\right)+m_{\phi \phi}^{2} \operatorname{Tr}\left(i \tau_{2} \phi^{T} i \tau_{2} \phi\right)\right. \\
& \left.+m_{\chi \chi}^{2} \operatorname{Tr}\left(i \tau_{2} \chi^{T} i \tau_{2} \chi\right)+\text { h.c. }\right)+m_{3}^{2}\left|\Delta_{R}\right|^{2}+m_{4}^{2}\left|\delta_{R}\right|^{2}-\left(m_{\Delta \delta}^{2} \operatorname{Tr} \Delta_{R} \delta_{R}+\text { h.c. }\right) \\
& +m_{5}^{2}\left|\Delta_{L}\right|^{2}+m_{6}^{2}\left|\delta_{L}\right|^{2}-\left(m_{\Delta \delta}{ }^{2} \operatorname{Tr} \Delta_{L} \delta_{L}+h . c .\right)+m_{7}^{2}\left|L^{c}\right|^{2}+m_{8}^{2}|L|^{2} \\
& +\left(L^{T} i \tau_{2}\left(A_{\phi} \phi+A_{\chi} \chi\right) L^{c}+A_{\Delta_{R}} L^{c T} i \tau_{2} \Delta_{R} L^{c}+A_{\delta_{L}} L^{T} i \tau_{2} \delta_{L} L+\text { h.c. }\right) \\
& +m_{9}^{2}|Q|^{2}+m_{10}^{2}\left|Q^{c}\right|^{2}+\left(Q^{T} i \tau_{2}\left(B_{\phi} \Phi+B_{\chi} \chi\right) Q^{c}+\text { h.c. }\right) .
\end{aligned}
$$

From (2), (4), (5) and (6) it is straightforward to derive the mass matrix for the CPeven neutral scalars, whose eigenvalues will provide the masses of the physical scalar Higgs particles. We shall not write the full $10 \times 10$ mass matrix [26] here, since for the specific purpose of the determination of a general bound on the lightest Higgs mass, the problem is much simpler. A known property of any Hermitian matrix is that its minimum eigenvalue must be smaller than that of its upper left corner $2 \times 2$ submatrix. Using this fact, and calling $m_{i j}$ the matrix elements of the CP-even neutral Higgs mass squared matrix, we can write the following rigorous bound on the squared mass $\left(m_{h}^{2}\right)$ of the lightest Higgs boson:

$$
m_{h}^{2} \leq \frac{m_{11}^{2}+m_{22}^{2}}{2}\left[1-\sqrt{1-4 \frac{m_{11}^{2} m_{22}^{2}-m_{12}^{4}}{m_{11}^{2}+m_{22}^{2}}}\right] .
$$

We shall choose a basis for the Higgs mass matrix such that the first two indices correspond to $\left(\Phi_{1}^{0}, \chi_{2}^{0}\right)$. Starting from the expression (2)-(6) for the potential and imposing the minimization conditions $\partial V / \partial \Phi_{1}^{0}=\partial V / \partial \chi_{2}^{0}=0$, we obtain the 
following expression for $m_{11}^{2}, m_{22}^{2}, m_{12}^{2}$ (from now on we will assume that only one $\mathrm{R}$-parity violating vacuum expectation value, namely $\left.\sigma_{R} \neq 0\right)$ :

$$
\begin{aligned}
& m_{11}^{2}=-m_{\Phi \chi}^{2} \frac{\kappa_{2}}{\kappa_{1}}+\frac{1}{2}\left(g_{L}^{2}+g_{R}^{2}\right) \kappa_{1}^{2}, \\
& m_{22}^{2}=-m_{\Phi \chi}^{2} \frac{\kappa_{1}}{\kappa_{2}}+\frac{1}{2}\left(g_{L}^{2}+g_{R}^{2}\right) \kappa_{2}^{2}, \\
& m_{12}^{2}=m_{\Phi \chi}^{2}-\frac{1}{2}\left(g_{L}^{2}+g_{R}^{2}\right) \kappa_{1} \kappa_{2} .
\end{aligned}
$$

From eqs. (7)-(8), we immediately obtain the upper bound on the lightest Higgs boson mass in the left-right supersymmetric model:

$$
m_{h}^{2} \leq \frac{1}{2}\left(g_{L}^{2}+g_{R}^{2}\right)\left(\kappa_{1}^{2}+\kappa_{2}^{2}\right) \cos ^{2} 2 \beta=\left(1+\frac{g_{R}^{2}}{g_{L}^{2}}\right) m_{W_{L}}^{2} \cos ^{2} 2 \beta,
$$

where $\tan \beta=\kappa_{2} / \kappa_{1}$. We note that the upper bound (9) is independent both of the supersymmetry breaking parameters (as in the case of supersymmetric models based on $\left.S U(2)_{L} \times U(1)_{Y}\right)$ and of the $S U(2)_{R}$ breaking scale, which, a priori can be very large. It is also independent of the $R$-parity breaking vacuum expectation value $\sigma_{R}$. The upper bound is controlled by the weak scale and the dimensionless gauge couplings $\left(g_{L}\right.$ and $\left.g_{R}\right)$. S Since the right-handed gauge coupling $g_{R}$ is not known, the upper bound on the right-hand side of (9) comes from the requirement that the left-right supersymmetric model remains perturbative below some scale $\Lambda$. In order to implement this requirement we need to solve the renormalization group equations (RGE's) for the gauge couplings of the theory. We do not require that the gauge couplings unify at some scale.

In the left-right supersymmetric theory defined by the superpotential (11), the one-loop RGE's for the $S U(2)_{L} \times S U(2)_{R} \times U(1)_{B-L}$ gauge couplings can be written as

$$
\begin{aligned}
16 \pi^{2} \frac{d g_{L, R}}{d t} & =6 g_{L, R}^{3}, \\
16 \pi^{2} \frac{d g_{B-L}}{d t} & =16 g_{B-L}^{3} .
\end{aligned}
$$

\footnotetext{
${ }^{2}$ Even if we do not neglect $\kappa_{1}^{\prime}, \kappa_{2}^{\prime}$ and $\sigma_{L}$, the upper bound on the lightest Higgs mass does not depend either on the supersymmetry breaking scale, or right-handed breaking scale, or $\sigma_{R}$ [26], although it depends on $\sigma_{L}$. However, since $\sigma_{L}$ contributes to the lighter $W$ mass, it is at most of the order of weak scale.
} 


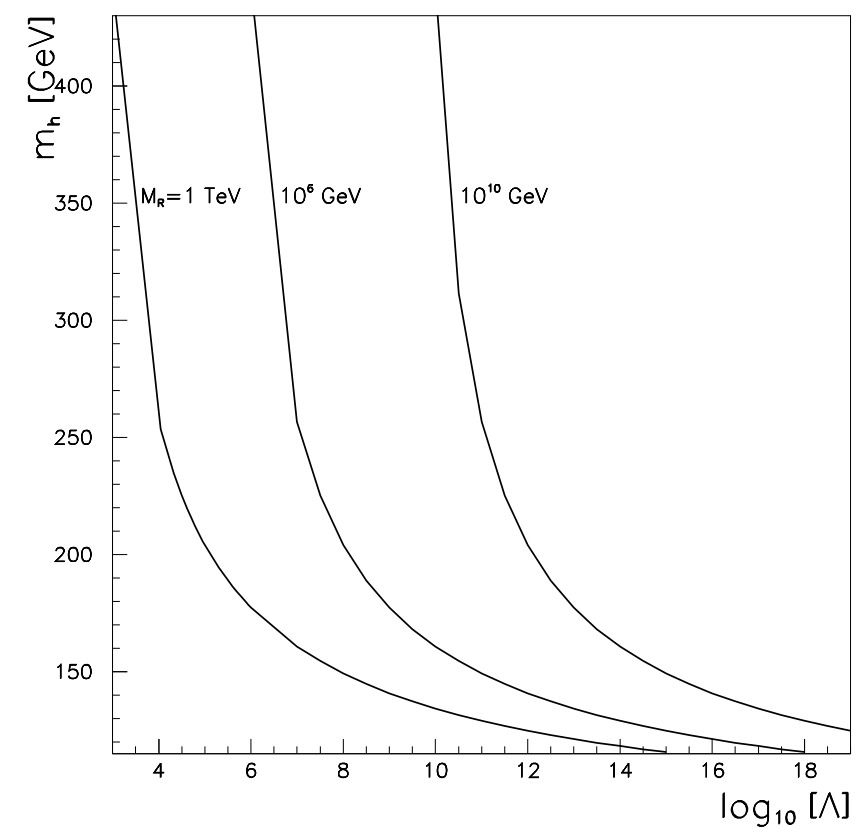

Figure 1: The tree level upper bound on the lightest Higgs mass as a function of the scale $\Lambda$ upto which the $g_{R}$ coupling remains perturbative. The plotted $S U(2)_{R} \times$ $U(1)_{B-L}$ breaking scales are $M_{R}=1 \mathrm{TeV}, 10^{6} \mathrm{GeV}$ and $10^{10} \mathrm{GeV}$.

Requiring that $g_{R}$ remains perturbative upto a scale $\Lambda\left(g_{i}^{2}\left(Q^{2}\right) / 4 \pi \leq 1\right.$ for $Q^{2} \leq \Lambda^{2}$, where the equality holds for $Q^{2}=\Lambda^{2}$ ), we obtain the upper bound on the lightest Higgs mass as shown in Fig. 1 for different values of $\Lambda$ ranging from $\Lambda=1 \mathrm{TeV}$ to $\Lambda=10^{19} \mathrm{GeV}$ and $S U(2)_{R} \times U(1)_{B-L}$ breaking scales $M_{R}=1 \mathrm{TeV}, 10^{6} \mathrm{GeV}$ and $10^{10} \mathrm{GeV}$, respectively. The gauge couplings $g_{L}, g_{R}$ and $g_{B-L}$ are connected by the weak mixing angle, $\tan \theta_{W}=g^{\prime} / g_{L}=g_{R} g_{B-L} / g_{L} \sqrt{g_{R}^{2}+g_{B-L}^{2}}$. We have checked that for the values used, also $g_{B-L}$ remains perturbative upto the scale $\Lambda$. The coupling $g_{R}$ has a lower limit from $\sin ^{2} \theta_{W}=e^{2} / g_{L}^{2} \simeq 0.23$, namely $g_{R} \geq 0.55 g_{L}$ [27], which is also fulfilled in Fig.1. Due to the lower limit on $g_{R}$, the smallest upper bound is given by $m_{h} \lesssim 92 \mathrm{GeV} \times|\cos 2 \beta|$. Since the bound (9) increases with increasing $g_{R}$, it becomes less restrictive for smaller values of $\Lambda$ or larger values of $M_{R}$. If the difference between $\Lambda$ and $M_{R}$ is larger than two orders of magnitude, the tree-level 
upper bound remains below $\sim 205 \mathrm{GeV}$. When $\Lambda=M_{R}$ and hence $g_{R}^{2}\left(\Lambda^{2}\right)=4 \pi$, the bound is at its largest, namely $m_{h} \lesssim 446 \mathrm{GeV}$.

We now proceed to the calculation of dominant one-loop radiative corrections to the upper bound (9) on the lightest Higgs boson mass in SUSYLR model. We shall use the method of one-loop effective potential [28] for the calculation of radiative corrections, where the effective potential may be expressed as the sum of tree-level potential plus a correction coming from the sum of one-loop diagrams with external lines having zero momenta,

$$
V_{1-\text { loop }}=V_{\text {tree }}+\Delta V_{1}
$$

where $V_{\text {tree }}$ is the tree level potential (2) evaluated at the appropriate running scale $Q$, and $\Delta V_{1}$ is the one-loop correction given by

$$
\Delta V_{1}=\frac{1}{64 \pi^{2}} \sum_{i}(-1)^{2 J_{i}}\left(2 J_{i}+1\right) m_{i}^{4}\left(\ln \frac{m_{i}^{2}}{Q^{2}}-\frac{3}{2}\right) .
$$

In (12), $m_{i}$ is the mass of the $i$ th particle with spin $J_{i}$ in the appropriate background. The dominant contribution to (12) arises from the top-stop $(t-\tilde{t})$ and bottomsbottom $(b-\tilde{b})$ systems. For the degenerate stop case the one-loop correction is given by

$$
\begin{aligned}
\Delta V_{1}= & \frac{3}{16 \pi^{2}}\left[\left(\tilde{m}^{2}+h_{t}^{2}\left|\kappa_{2}\right|^{2}\right)^{2}\left(\ln \frac{\tilde{m}^{2}+h_{t}^{2}\left|\kappa_{2}\right|^{2}}{Q^{2}}-\frac{3}{2}\right)\right. \\
& \left.-h_{t}^{4}\left|\kappa_{2}\right|^{4}\left(\ln \frac{h_{t}^{2}\left|\kappa_{2}\right|^{2}}{Q^{2}}-\frac{3}{2}\right)\right],
\end{aligned}
$$

with an analogous expression for the sbottom case. In (13) $\tilde{m}^{2}$ is the SUSY breaking mass for the squarks, $Q$ is the renormalization scale, and $h_{t}$ is the top Yukawa coupling $\left(h_{t}=\left(h_{\chi Q}\right)_{33}\right.$, see (1i)). In the case with a large left-right squark mixing the formula (13) should be extended appropriately [26]. In numerical calculations, we shall use the full formula which includes the left-right mixing in stop and sbottom mass matrices.

Using (13), one can derive the radiatively corrected upper bound on the mass of the lightest CP-even Higgs boson in the left-right supersymmetric model: 


$$
m_{h}^{2} \leq \frac{1}{2}\left[\left(g_{L}^{2}+g_{R}^{2}\right)\left(\kappa_{1}^{2}+\kappa_{2}^{2}\right) \cos ^{2} 2 \beta+G\left(\Delta_{11} \cos ^{2} \beta+\Delta_{22} \sin ^{2} \beta+\Delta_{12} \cos 2 \beta\right)\right]
$$

where $G=3 g_{L}^{2} /\left(8 \pi^{2} m_{W}^{2}\right)$, and $\Delta_{i j}(i, j=1,2)$, which signify radiative corrections, are complicated functions of the parameters of the model [26], and are similar in structure to the corresponding quantities in the models based on $S U(2)_{L} \times U(1)_{Y}$ gauge group [29]. For the degenerate stop and sbottom case, they reduce to

$$
\Delta_{11}=\frac{m_{b}^{4}}{\cos ^{2} \beta} \ln \left(\frac{m_{\tilde{b}_{1}}^{2} m_{\tilde{b}_{2}}^{2}}{m_{b}^{4}}\right), \Delta_{22}=\frac{m_{t}^{4}}{\sin ^{2} \beta} \ln \left(\frac{m_{\tilde{t}_{1}}^{2} m_{\tilde{t}_{2}}^{2}}{m_{t}^{4}}\right),
$$

with $\Delta_{12}=0$, i.e. the same as in MSSM. In Fig. 2, we have plotted the upper bound (14) as a function of top quark mass in the range $150<m_{t}<200 \mathrm{GeV}$, which subsumes the recent direct measurement (CDF and D0 combined) from the Tevatron $p \bar{p}$ collider of $m_{t}=175 \pm 6 \mathrm{GeV}$ [30.

In Fig. 2 we have taken the soft supersymmetry breaking trilinear couplings to be $A_{t}=A_{b}=1 \mathrm{TeV}$, where $A_{t}=\left(B_{\Phi} h_{\Phi Q}^{-1}\right)_{33}, A_{b}=\left(B_{\chi} h_{\chi Q}^{-1}\right)_{33}$. The solid line corresponds to $S U(2)_{R} \times U(1)_{B-L}$ breaking scale $10 \mathrm{TeV}$ and dashed one to $10^{10} \mathrm{GeV}$. The bound on the lightest Higgs mass becomes less restrictive for larger $S U(2)_{R}$ breaking scales. In Fig. 2 a) we have taken $\mu_{1}=\mu_{1}^{\prime}=\mu_{1}^{\prime \prime}=0$. The MSSM limit with $\mu=0$ and $\tan \beta=20$ is plotted as dotted line. In all cases $\Lambda=10^{16}$ $\mathrm{GeV}$. The upper bound increases with increasing $M_{R}$ scale. For $M_{R}=10 \mathrm{TeV}$ and $m_{\text {top }}=175 \mathrm{GeV}$, the bound remains below $155 \mathrm{GeV}$ while for $M_{R}=10^{10} \mathrm{GeV}$ it remains below $175 \mathrm{GeV}$. In Figures $2 \mathrm{~b}$ ) and c) the dependence on $\mu_{1}, \mu_{1}^{\prime}$ and $\mu_{1}^{\prime \prime}$ is shown. The dependence on $\mu$-type parameters is enhanced compared to the MSSM expressions [29], since $-\mu \tan \beta$ is replaced by $\left(\mu_{1}+2 \mu_{1}^{\prime \prime} \frac{m_{t}}{m_{b}} \cot \beta\right) \tan \beta$. Except for large $\mu_{1}, \mu_{1}^{\prime}, \mu_{1}^{\prime \prime}$, it is seen that the mass limits are somewhat higher in SUSYLR than in the MSSM. Compared to the limits from models with gauge singlets [31, 32, 11], one finds that, depending on the unknown couplings, the limit may be smaller or larger.

To conclude we have obtained an upper bound on the lightest Higgs boson mass in the supersymmetric left-right model, and have shown that it does not depend on the soft supersymmetry breaking parameters or potentially large $S U(2)_{R} \times U(1)_{B-L}$ 
breaking scales. Furthermore it does not depend on the vacuum expectation value of the right-handed sneutrino. The tree-level bound, however, can be considerably larger than the corresponding bound in MSSM, if the difference between the high scale $\Lambda$ and the intermediate scale $M_{R}$ is small. The radiative corrections to the upper bound from top-stop and bottom-sbottom sector are sizable and of the same form as in the MSSM.

\section{Acknowledgements}

One of us (PNP) would like to thank the Helsinki Institute of Physics for hospitality while this work was completed. The work of PNP is supported by the Department of Atomic Energy Project No.37/14/95-R \& D-II/663.

\section{References}

[1] For reviews of supersymmetry, see H. Haber and G. L. Kane, Phys. Rep. 117 (1985) 76; H.P. Nilles, Phys. Rep. 110 (1984) 1; A. Chamseddine, P. Nath, and R. Arnowitt, Applied $N=1$ Supersymmetry (World Scientific, Singapore, 1984).

[2] K. Inoue, A. Kakuto, H. Komatsu and S. Takeshita, Prog. Theor. Phys. 67 (1982) 1889; R. Flores and M. Sher, Ann. Phys. (NY)148 (1983) 95.

[3] Y. Okada, M. Yamaguchi and T. Yanagida, Prog. Theor. Phys.85 (1991) 1; J. Ellis, G. Ridolfi and F. Zwirner, Phys. Lett. B257 (1991) 83; H.E. Haber and R. Hempfling, Phys. Rev. Lett. 66 (1991) 1215; R. Barbieri, M. Frigeni and F. Caravaglios, Phys. Lett. 258 (1991) 167.

[4] J. Rosiek and A. Sopczak, Phys. Lett. B341 (1995) 419.

[5] J. Ellis, J.F. Gunion, H.E. Haber, L. Roszkowski and F. Zwirner, Phys. Rev. D39 (1989) 844; M. Drees, Int. J. Mod. Phys. A4 (1989) 3635. 
[6] P. Binetruy and C.A. Savoy, Phys. Lett. B277 (1992) 453; T. Moroi and Y. Okada, Phys. Lett. B295 (1992) 73.

[7] P.N. Pandita, Phys. Lett. B318 (1993) 271; Z. Phys. C59 (1993) 575.

[8] T. Elliot, S.F. King and P.L. White, Phys. Lett. B305 (1993) 71; Phys. Rev. D49 (1994) 4435.

[9] U. Ellwanger, Phys. Lett. B303 (1993) 271; U. Ellwanger, M. Lindner, Phys. Lett. B301 (1993) 365.

[10] G.L. Kane, C. Kolda and J. Wells, Phys. Rev. Lett. 70 (1993) 2606; J.R. Espinosa and M. Quiros, Phys. Lett. B279 (1992) 92; Phys. Lett. B302 (1993) 51.

[11] J.R. Espinosa, Phys. Lett. B 353 (1995) 243; D. Comelli and J.R. Espinosa, Phys. Lett. B 388 (1996) 793.

[12] G. Farrar and P. Fayet, Phys. Lett. B76 (1978) 575.

[13] S. Dimopoulos and H. Georgi, Nucl. Phys. B193 (1981) 150; S. Weinberg, Phys. Rev. D26 (1982) 287; N. Sakai and T. Yanagida, Nucl. Phys. B197 (1982) 533.

[14] S. Giddings and A. Strominger, Nucl. Phys. B307 (1988) 854; S. Coleman, Nucl. Phys. B310 (1988) 643; J. Preskill and L.M. Krauss, Nucl. Phys. B341 (1990) 50; R. Holman et al., Phys. Lett. B282 (1992) 132; M. Kamionkowski and J. March-Russell, Phys. Lett. B282 (1992) 137.

[15] F. Zwirner, Phys. Lett. B132 (1983) 103; L.J. Hall and M. Suzuki, Nucl. Phys. B231 (1984) 419; I.H. Lee, Nucl. Phys. B246 (1984) 120; S. Dawson, Nucl. Phys. B261 (1985) 297; R. Barbieri and A. Masiero Nucl. Phys. B267 (1986) 679; S. Dimopoulos and L.J. Hall, Phys. Lett. B207 (1987) 210; V. Barger, G.F. Giudice, and T. Han, Phys. Rev. D40 (1989) 2987; H. Dreiner and G.G. Ross, Nucl. Phys. B365 (1991) 597.

[16] R.N. Mohapatra, Phys. Rev. D34 (1986) 3457; A. Font, L.E. Ibanez, and F. Quevedo, Phys. Lett. B228 (1989) 79. 
[17] S.P. Martin, Phys. Rev. D46 (1992) 2769; Phys. Rev. D54 (1996) 2340.

[18] M. Cvetic and J.C. Pati, Phys. Lett. B135 (1984) 57.

[19] R.M. Francis, M. Frank and C.S. Kalman, Phys. Rev. D43 (1991) 2369; R.M. Francis, C.S. Kalman, and H.N. Saif, Z. Phys. C59 (1993) 655.

[20] R. Kuchimanchi and R.N. Mohapatra, Phys. Rev. D48 (1993) 4352.

[21] K. Huitu, J. Maalampi, and M. Raidal, Nucl. Phys. B420 (1994)449; Phys. Lett. B320 (1994) 60.

[22] K. Huitu and J. Maalampi, Phys. Lett. B344 (1995) 217.

[23] K. Huitu, P.N. Pandita, K. Puolamäki, Helsinki Institute of Physics preprint HIP-1997-06/TH, hep-ph/9702415.

[24] Particle Data Group, Review of Particle Properties, Phys. Rev. D54 (1996) 1.

[25] R. Kuchimanchi and R.N. Mohapatra, Phys. Rev. Lett. 75 (1995) 3989.

[26] K. Huitu, P.N. Pandita and K. Puolamäki, in preparation.

[27] M. Cvetic, P. Langacker, Phys. Rev. Lett. 68 (1992) 2871.

[28] S. Coleman and E.J. Weinberg, Phys. Rev. D7 (1973) 1888.

[29] J. Ellis, G. Ridolfi, F. Zwirner, Phys. Lett. B 262 (1991) 477; Phys. Lett. B 257 (1991) 83.

[30] P. Grannis, presented at the International Conference on Higgs Energy Physics, Warsaw (1996).

[31] P.N. Pandita, Phys. Lett. B 318 (1993) 338; Z. Phys. C 59 (1993) 575.

[32] J. Kamoshita, Y. Okada, M. Tanaka, Phys. Lett. B 328 (1994) 67. 

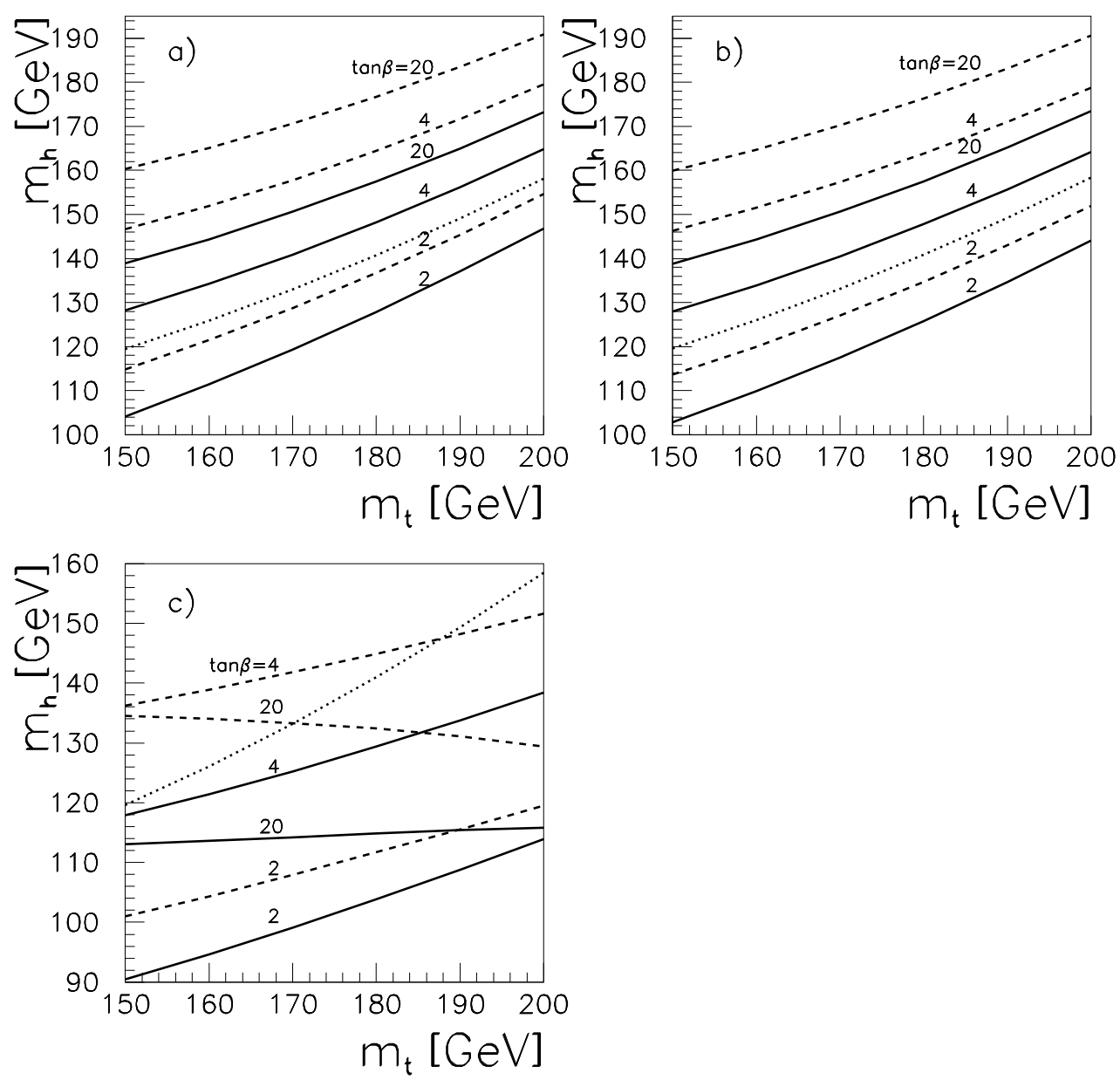

Figure 2: The radiatively corrected upper limit on the mass of the lightest Higgs boson as a function of $m_{t}$ with $\Lambda=10^{16} \mathrm{GeV}$ and $A_{t}=A_{b}=1=\mathrm{TeV}$. The solid line corresponds to the $S U(2)_{R}$ scale of $10 \mathrm{TeV}$ and the dashed line to the $S U(2)_{R}$ scale of $10^{10} \mathrm{GeV}$. The dotted curve corresponds to MSSM limit for $\tan \beta=20$ and $\mu=\mu_{1}$. In a) $\mu_{1}=\mu_{1}^{\prime}=\mu_{1}^{\prime \prime}=0$, in b) $\mu_{1}=\mu_{1}^{\prime}=\mu_{1}^{\prime \prime}=500 \mathrm{GeV}$, and in c) $\mu_{1}=\mu_{1}^{\prime}=\mu_{1}^{\prime \prime}=1000 \mathrm{GeV}$. 